\title{
Heat Shock Protein 60: Identification of an Undetected Allergen from Dermatophagoides farinae
}

\author{
Zongmin Liao ${ }^{1,2}$, Haijuan Liu ${ }^{3}$, Jian Liu ${ }^{4}$, Mingsheng Cai ${ }^{1}$, Tao Chen ${ }^{1}$, Qing Hong ${ }^{1}$, Xiaodong Luo ${ }^{1}$, \\ Xiaomin Li ${ }^{1}$, Xue Ding ${ }^{1}$, Haoxian Shen ${ }^{1}$, Daixiong Chen ${ }^{1, *}$ \\ ${ }^{1}$ Department of Pathogenic Biology and Immunology, Sino-French Hoffmann Institute, School of Basic Medical Science, Guangzhou \\ Medical University, Xinzao Town, Panyu, Guangzhou 511436, Guangdong, China \\ ${ }^{2}$ Department of Scientific Research and Education, Yuebei People's Hospital, Shaoguan 512026, Guangdong, China. \\ ${ }^{3}$ Department of Clinical Laboratory Sciences, Renmin Hospital, Hubei University of Medicine 442000, Hubei, China \\ ${ }^{4}$ Department of gynaecology, Yuebei People's Hospital, Shaoguan 512026, Guangdong, China. \\ "Corresponding author: Daixiong Chen. Department of Pathogenic Biology and Immunology, Sino-French Hoffmann Institute, School of Basic Medical \\ Science, Guangzhou Medical University, Xinzao Town, Panyu, Guangzhou 511436, Guangdong, China, Tel/Fax: +86-15989006019; \\ E-mail: daixiongchen@hotmail.com
}

Received: 16 Aug. 2016; Revised: 26 May 2018; Accepted: 28 May. 2018; Published online: 11 Aug. 2018

\begin{abstract}
Background: Recently, the incidence of allergic diseases has been on the rise; Dust mite is the major indoor allergen which needs a special consideration.

Objectives: This study was carried out to identify and investigate the molecular properties of a new allergen named Hsp60 and to afford a foundation for future research of the allergic diseases caused by Dermatophagoides farinae.

Materials and Methods: Using polymerase chain reaction (PCR) with degenerate primer, the cDNA of Dermatophagoides farinae Hsp60 was amplified and sequenced. Next, the cDNA fragment was cloned into the prokaryotic expression vector pET-32a for the expression of the Hsp60. Then, it was further characterized by Elisa and Western Blot analysis.

Results: The partial cDNA sequence of the Dermatophagoides farinae Hsp60 was determined, and the recombinant Hsp60 was successfully expressed in Escherichia coli BL21. ELISA and Western blot analysis showed that the recombinant protein could be specifically recognized by SIgE from sera of the Dermatophagoides farina-allergic patients.

Conclusions: Our group has, for the first time, demonstrated the fact that there is an Hsp60 family of Dermatophagoides farinae and analyzed the allergenicity of the Hsp60 with immunological method. These results provide a foundation for further allergological research of the Dermatophagoides farinae Hsp60.

Keywords: Dermatophagoides farinae; Expression; Hsp60
\end{abstract}

\section{Background}

In recent years, the incidence of allergic diseases has shown an upward trend, resulting in a global public health problem (1-4). As an inhalant allergen, the dust mite attracted great attention in allergological research (58). It was confirmed that dust mites Dermatophagoides farinae and Dermatophagoides pteronyssinus are the major indoor allergens that can cause bronchial asthma, allergic rhinitis, atopic dermatitis, and other allergic diseases (7-9).
The study of dust mite allergens is the basis for understanding the allergic diseases caused by dust mites. Each day, more and more new dust mite allergens are being identified $(10,11)$, greatly increasing our knowledge of dust mite allergens, promoting the levels of diagnosis, prevention, and treatment of the dust mite allergic diseases. On the basis of our previous study (12), 84 allergen proteins of $D$. farinae were identified using LC-MS/MS methods. These allergen proteins include some previously reported house dust

Copyright (C) 2017 The Author(s); Published by National Institute of Genetic Engineering and Biotechnology. This is an open access article, distributed under the terms of the Creative Commons Attribution-NonCommercial 4.0 International License (http://creativecommons.org/licenses/ by-nc/4.0/) which permits others to copy and redistribute material just in noncommercial usages, provided the original work is properly cited. 
mite allergens, many kinds of enzymes, translation elongation factor, ribosomal protein, outer membrane (lipo-) protein, keratin, glycine cleavage system protein, binding protein, heat shock protein, regulation protein, hypothetical protein, pol polyprotein, pol protein, and etc. As an inhalant allergen, the heat shock protein 60 (Hsp60) from $D$. farinae was further investigated to identify undetected $D$. farinae allergens.

\section{Objective}

In this study, to identify the new allergen the partial cDNA sequence of the $D$. farinae $\mathrm{Hsp} 60$ was determined. Moreover, the cDNA fragment was cloned and transformed into E. coli for the prokaryotic expression of the protein. The immunological characteristics of the expressed product were analyzed by ELISA and Western blot methods to offer a foundation for future investigation of the allergic diseases caused by Dermatophagoides farinae.

\section{Materials and Methods}

\subsection{Dust mite D. Farinae}

We used a vacuum cleaner (PHILPS FC6048, Qingdao co., LTD.) to collect samples of dust mites from the room and pillow of some students at the Guangzhou Medical University. A surgical mask was used to cover the vacuum tube. The surgical mask was replaced each time. The retrieved samples were placed on a plastic plate (KA061, $9 \mathrm{~cm})$, and the mites were detected with a zero brush under the body vision microscope (smz-168, Motic company). Dust mite $D$. farinae was cultured at a constant temperature $\left(27 \pm 0.5^{\circ} \mathrm{C}\right)$ and humidity (relative humidity of $75 \% \pm 1 \%$ ) in our laboratory and afterwards stored at $-80{ }^{\circ} \mathrm{C}$.

\subsection{Plasmid}

Prokaryotic expression vector pET-32a (+) was kindly provided by Professor Peng Lifei of Guangdong Medical College. $\mathrm{P}^{\mathrm{MD} 18-\mathrm{T}}$ vector, an effective vector for Cloning of PCR products (TA Cloning), was purchased from TaKaRa (Biotechnology, Dalian, China).

\subsection{Serum}

The hypersensitivity of patients to $D$. farina was determined with the Uni-CAP 100-automatic allergen detection system (Pharmacia, Sweden), and their sera were collected by the Guangzhou Institute of Respiratory Diseases and Kingmed Diagnostics Center. Serum samples from 50 patients, with levels of specific $\operatorname{IgE}$ (SIgE) against $D$. farina at 3 or more, were mixed and used in this study. The negative control sera lacking the SIgE were collected from the people with negative skin prick test results and without a history of allergy to house-dust mites.

\subsection{RNA Extraction and cDNA Synthesis}

The homogenate of 300 dust mites $D$. farinae was mixed in $1 \mathrm{ml}$ Trizol (Invitrogen, USA) and RNA was extracted according to the Trizol Reagent's instructions. Then the RNA was reverse-transcribed into cDNA with PrimeScript RT-PCR Kit (TaKaRa, Dalian, China).

\subsection{PCR Amplification}

On the basis of the characteristic and conservative sequences of the Hsp60 homologues of other species, the degenerate primers were designed. The upstream primer was 5 '-GATGAACCCNATGGAYYTNAARMGNGG-3', and the downstream primer was 5'-AGTC ACCAAATCCNGGNGCYTTNAC-3'. PCR amplifications were performed using the prepared cDNA as a template, and the following cycling parameters were used in the PCR reactions (BIO-RAD T100, USA): $94^{\circ} \mathrm{C}$ for $5 \mathrm{~min}, 30$ cycles of $94^{\circ} \mathrm{C}$ for $45 \mathrm{~s}$, $60{ }^{\circ} \mathrm{C}$ for $45 \mathrm{~s}$, and $72{ }^{\circ} \mathrm{C}$ for $90 \mathrm{~s}$, and $72{ }^{\circ} \mathrm{C}$ for $10 \mathrm{~min}$.

\subsection{Sequencing Analysis}

The PCR products were sequenced by Takara Biotechnology Companies (Dalian, China). To investigate homology, the acquired nucleotide sequence and the corresponding amino acid sequence were analyzed with BLAST (http://www.ncbi.nlm.nih.gov/).

\subsection{TA Cloning}

According to the sequencing result, specific PCR primers were designed. (Upstream primer was 5'-CGGGATCCCCAATGGATTTTAAACGCG-3' and downstream primer was 5'-CGCTCGAGTCCTGGTGCCTTCACG-3', underlines show the $B a m H I$ and $X h o$ Irestriction sites, respectively). Then PCR amplification of the D. farinae Hsp60 gene fragment was carried out, and the reaction conditions were $94^{\circ} \mathrm{C}$ for $5 \mathrm{~min}, 30$ cycles of $94^{\circ} \mathrm{C}$ for $45 \mathrm{~s}, 56 \mathrm{v}$ for $45 \mathrm{~s}, 72^{\circ} \mathrm{C}$ for $90 \mathrm{~s}$, and $72{ }^{\circ} \mathrm{C}$ for $10 \mathrm{~min}$. PCR products were purified by an agarose gel DNA extraction kit (Tiangen Biotech, Beijing, China) and subsequently were inserted into the $\mathrm{P}^{\mathrm{MD} 18-\mathrm{T}}$ vector to construct $\mathrm{P}^{\mathrm{MD} 18-\mathrm{T}}$ Hsp60. The recombinant plasmid was identified with PCR amplification and restriction enzymes digestion.

\subsection{Subcloning}

The plasmid $\mathrm{P}^{\mathrm{MD} 18-\mathrm{T}}$-Hsp60 was digested with BamH I and Xho I (TaKaRa, Dalian, China). 
Afterwards, the Hsp60 fragment was subcloned into the corresponding digested pET-32a(+) vector to construct pET-32a(+)-Hsp60 (containing His tag). The recombinant plasmid was transformed into $E$. coli $\mathrm{DH} 5 \alpha$, and was identified with the BamHI and the XhoI restriction enzymes. The identified plasmid pET-32a $(+)-H s p 60$ was then transformed into E. coli BL21 for expression.

\subsection{Protein Expression}

The expression of His-tagged recombinant Hsp60 in transformant $E$. coli BL21 was induced by adding isopropyl 1-thio- $\beta$-D-galactopyranoside With IPTG (Invitrogen, USA) (final concentration $=1 \mathrm{mmol} . \mathrm{L}^{-1}$ ) at $37^{\circ} \mathrm{C}$ for two hours, and the expression products were analyzed by SDS-PAGE as described by Sambrook (13)

\subsection{Purification of the Recombinant Protein Hsp60}

The soluble recombinant Hsp60 protein was purified by $\mathrm{Ni}^{2+}$-NTA agarose (Qiagen) according to the manufacturer's instructions. A glass column (capacity $=20 \mathrm{~mL}$ ) was packed with $\mathrm{Ni}^{2+}$-NTA resin matrix. The column was equilibrated with 4 bed volumes of IMAC buffer (20 mM Tris- $\mathrm{HCl}, \mathrm{pH} 8.0,500 \mathrm{mM} \mathrm{NaCl}, 0.5 \mathrm{mM}$ PMSF, and $10 \mathrm{mM}$ imidazole). The chromatography flow rate was $0.5 \mathrm{~mL} \cdot \mathrm{min}^{-1}$. The supernatant was loaded onto the $\mathrm{Ni}^{2+}$-NTA agarose resin column, preequilibrated with IMAC buffer. The column was washed successively with 3 bed volumes of IMAC buffer and 5 bed volumes of IMAC buffer containing $20 \mathrm{mM}$ imidazole. The protein impurities devoid of histidine tails were removed from the agarose resin at a flow rate of $2.0 \mathrm{~mL} \cdot \mathrm{min}^{-1}$ at $4{ }^{\circ} \mathrm{C}$. The fusion protein was eluted with IMAC buffer containing $8 \mathrm{M}$ urea and $100 \mathrm{mM}$ imidazole at a flow rate of $1.0 \mathrm{~mL} \cdot \mathrm{min}^{-1}$ at 4 ${ }^{\circ} \mathrm{C}$ Five-milliliter fractions were collected and analyzed by SDS-PAGE to identify the fusion protein and to assess the level of homogeneity. The purified protein was then dialyzed in PBS (pH 7.2) and stored at $-20{ }^{\circ} \mathrm{C}$ for further studies.

\subsection{ELISA}

In ELISA assay, microtiter plates were coated with the purified recombinant protein $\left(20 \mu \mathrm{g} \cdot \mathrm{mL}^{-1}, 100 \mu \mathrm{L}\right.$ per well). The dilution of the sera from the patients hypersensitive to $D$. farina was 1:100, and 1:3000 for the goat anti-human IgE-HRP antibody (KPL Inc. USA). The OD values of the samples were measured at $450 \mathrm{~nm}$ with a microplate reader (Thermo Multiskan GO, USA). All the experiments were carried out at least three times and the average OD values from three wells were collected.

\subsection{Western Blot Analysis}

The purified recombinant Hsp60 was transferred onto PVDF membrane according to the BIO-RAD TransBlot SD operations guide. The sera from the patients hypersensitive to $D$. farina were diluted 25 -fold and were used to react with the recombinant protein at $4{ }^{\circ} \mathrm{C}$ overnight. Goat anti-human IgE-HRP (KPL Inc. USA) was diluted at a ratio of 1:2500, then reacted with the membrane for $1 \mathrm{~h}$, and finally the results were visualized using the ECL chemiluminescence method.

\section{Results}

\section{1. PCR Amplification and cDNA Sequencing}

Using the degenerate primers, a product about 500 bp was successfully amplified by PCR. As shown in Figure 1, the PCR product was sequenced. BLAST indicated that the cDNA sequence was $90 \%$ similar to the Hsp60 of bacterium Variovorax, and the potential amino acid sequence showed $94 \%$ similarity to the Hsp60 of bacterium Acidovorax avenae.

\subsection{Construction of a Plasmid Expressing pET-32a(+)- Hsp60 Recombinant Protein}

Specific PCR primers with restriction sites $B a m \mathrm{H}$ I and Xho I were used to amplify the Hsp60 gene fragment of $D$. farinae, and the result (Fig. 2A) showed that the size (about $520 \mathrm{bp}$ ) of the amplified product is consistent with our expectation. The results of PCR amplification and the BamHI and the $X h o$ I restriction enzymes digestion of $\mathrm{P}^{\mathrm{MD} 18-}$ ${ }^{\mathrm{T}}$-Hsp60 are shown in Figure. 2B, suggesting that the target gene was successfully cloned into the vector. Subsequently, the Hsp60 gene fragment in recombinant plasmid $\mathrm{P}^{\mathrm{MD} 18-\mathrm{T}}-\mathrm{Hsp} 60$ was subcloned into plasmid pET-32a $(+)$, and Figure. 2C shows that the recombinant plasmid pET-32a(+)-Hsp60 was successfully constructed.

\subsection{Expression and Solubility Analysis of the Recombinant Protein}

As shown in Figure. 3A, in comparison with the control, pET-32a (+)-Hsp60 could express a protein about $37 \mathrm{KD}$, which is consistent with putative molecular mass of the fusion protein. In addition, the expression of the recombinant protein could be found in the supernatant and pellet of culture medium, and the expression of the recombinant protein in the supernatant demonstrates higher water-solubility (Fig. 3B).

\subsection{Purification of the Recombinant Protein}

The result for the purification of the soluble Hsp60 
A

CCAATGGATT TTAAACGCGG CATCGACAAG GCGGTCACOG CCCTGGTCGC CGAGCTGAaG 60 AAGGCCTCCA AGGCCACCAC CACCTCCAAG GAAATCGCCC AGGTCGGCAC CATCTCGGCC 120 AACAGCGACT CCGACGTCGG CCAGATCATC GCTGACGCGA TGGACAAGGT CGGCAAGGAA 180 GGCGTCATCA CCGTCGAAGA CGGCAAGAGC CTGAACAACG AGCTTGACGT CGTCGAAGGC 240 ATGCAGTTCG ACCGCGGCTA CCTGTCGCCC TACTTCATCA ACAACCCGGA AAAGCAAGCC 300 GCGATCCTGG ACAACCCGTC TGTCCTGCTG TTCGACAAGA AGATCAGCAA CATCCGCGAC 360 CTGCTGCCCA OGCTGGAAGC CGTGGCCAAG GCCGGCOGCC OGCTGCTGAT CATTGCCGAG 420 GAAGTCGAAG GCGAAGCCCT CGCGACCCTG GTGGTCAACA CCATCCGCGG CATCCTGAAG 480 GTTGTCGCCG TGAAGGCACC AGGA

B

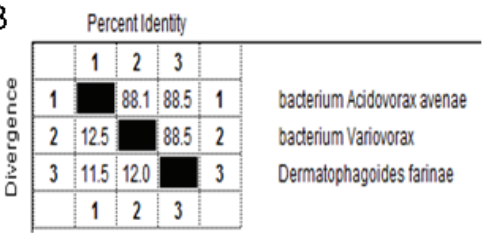

C
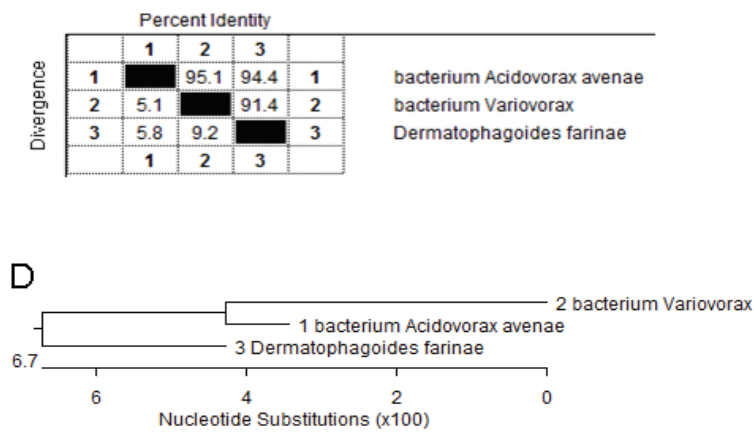

fusion protein is shown in Figure 4, which implies the recombinant protein was purified effectively.

\subsection{ELISA}

The average OD values of the patient sera, the negative control sera, and the blank controls were $0.383,0.126$, and 0.079 , respectively. The OD values of the patient sera is $>2.1$-fold of the negative control sera, which proves that the recombinant protein can react with SIgE in the patient sera.

\subsection{Western Blot Analysis}

The results for the Western blot analysis of the recombinant Hsp60 are shown in Figure 5, which shows that the recombinant protein could be specifically recognized by SIgE in the patient sera.

Figure 1. Sequencing result, nucleic acid sequence alignments, AA sequence alignments and evolutionary relationships of the $\mathrm{D}$. farinae Hsp60 with its reference species. (A) Sequencing result; (B) Multiple nucleic acid sequence alignments; (C) Multiple aa sequence alignments; (D) Evolutionary relationships. The alignments and phylogenetic tree were generated using the Megalign program in LASERGENE (DNAStar 7.0) with the Clustal V method, and sequence distance was calculated using weight matrix PAM250. Gaps were introduced by the alignment program to maximize the homology.
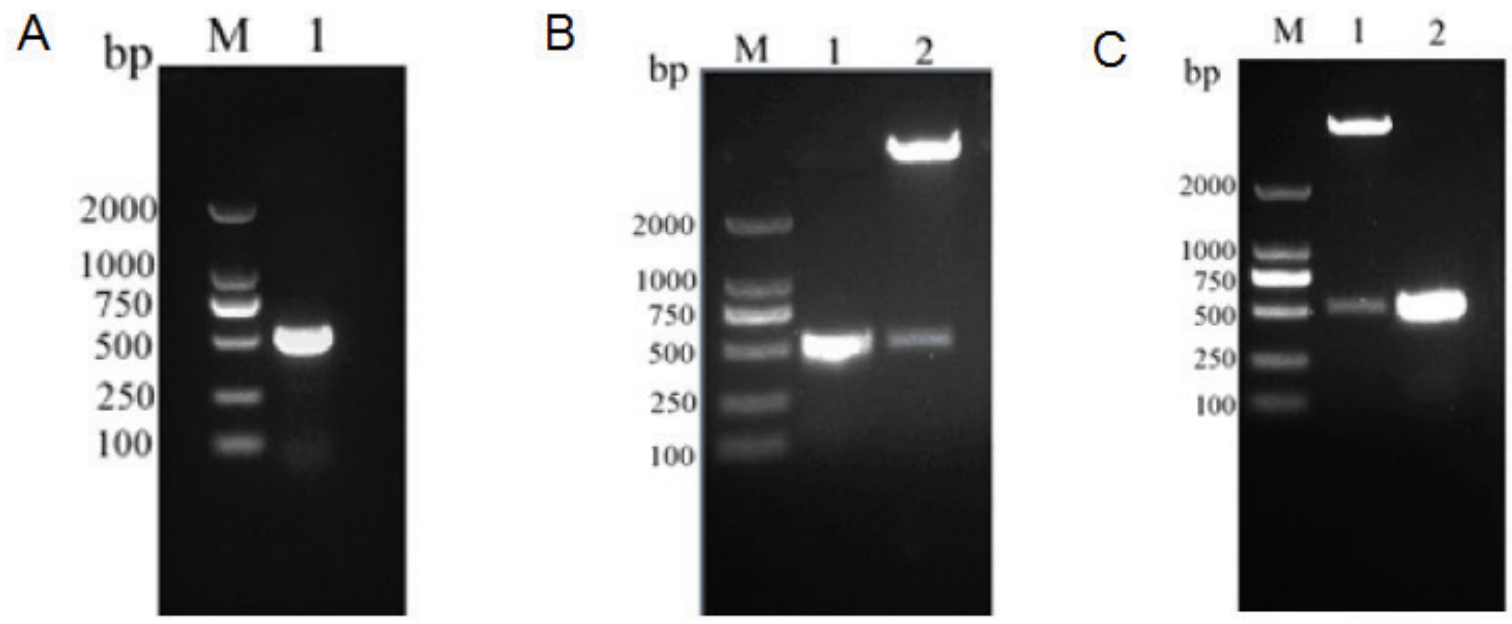

Figure 2. Cloning and verification of plasmid pET-32a(+)-Hsp60. (A) PCR amplification of D. farinae Hsp60. Lane M: DNA Marker; Lane 1: PCR product. (B) Identification of recombinant plasmid PMD18-THsp60. Lane M: DNA Marker; Lane 1: PCR product of the recombinant plasmids PMD18-T-Hsp60; Lane 2: Recombinant plasmid PMD18-T-Hsp60 digested with BamHIand XhoI. (C) Identification of recombinant plasmids pET-32a(+)-Hsp60. Lane M: DNA Marker; Lane 1: Recombinant plasmid pET-32a(+)-Hsp60 digested with BamHIand XhoI; Lane 2: PCR product of recombinant plasmid pET-32a(+)-Hsp60. 
A

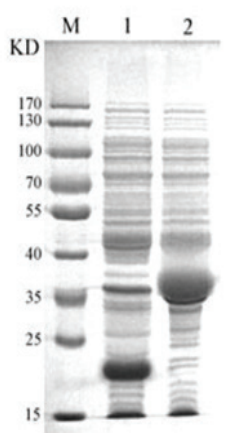

B

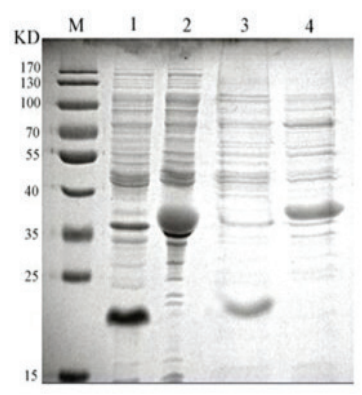

Figure 3.Expression and solubility analysis of the recombinant protein. (A) SDS-PAGE analysis of the expression of pET32a(+)-Hsp60. Lane M: molecular mass standards; Lane 1: Expression product of pET-32a $(+)($ control); Lane 2: Expression product of pET-32a(+)-Hsp60. (B) Solubility analysis of pET-32a $(+)-H s p 60$ expression product. Lane M: molecular mass standards; Lane 1: Expression product of pET-32a $(+)$ (pellet); Lane 2: Insoluble expression product of pET-32a $(+)-H s p 60($ pellet); Lane 3: Expression product of pET-32a $(+)($ supernatant); Lane 4: Soluble expression product of pET-32a(+)-Hsp60 (supernatant).

\section{Discussion}

The heat shock proteins (HSPs) are important for the growth and the development of prokaryotes and eukaryotes. It is well known when organisms are stimulated by physical, chemical, and pathological factors, the expression levels of the HSPs increase. It has been reported that the HSPs can be recognized by the immune system, and is involved in the antigen processing and presenting (14). Moreover, evidently the HSPs of some parasites could induce immunological response in the host, indicating their function as

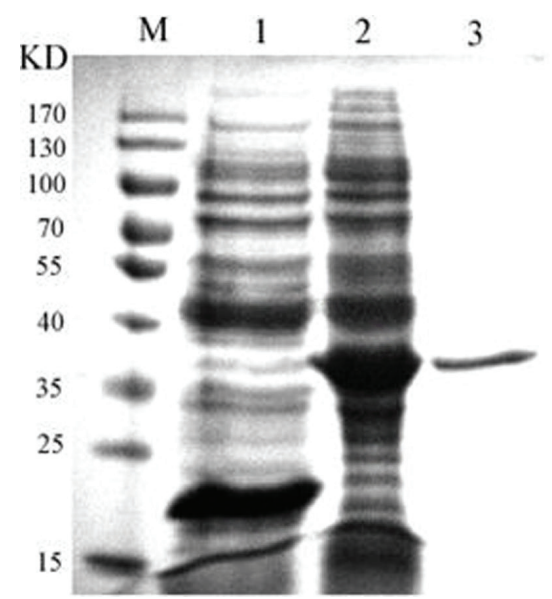

Figure 4. Purification of the recombinant protein Lane M: molecular mass standards; Lane 1: Expression product of pET-32a (+); Lane 2,3: unpurified and purified expression products of $\mathrm{pET}-32 \mathrm{a}(+)$-Hsp60, respectively.

antigen $(15,16)$. In addition, it has been confirmed that the Hsp70 of $D$. farinae may function as an allergen (17). Based on the results of our previous study (12), we suspect that $D$. farinae Hsp60 is also an allergen. In combination with another research conducted by our group (18), we have demonstrated the existence of a Hsp60 family of $D$. farinae .

In this study, the partial cDNA sequence of the $D$. farinae Hsp60 was successfully identified and it was efficiently expressed in the prokaryotic expression system. Moreover, ELISA and western blot analysis revealed that the expressed recombinant protein could be specifically recognized by SIgE in sera of the $D$. farinae allergic patients. The results suggest that the Hsp60 may be an undetected D. farinae allergen.

Currently, using the patients' sera as a probe, the immunological screening of the cDNA library is a routine method to identify and discover new dust mite allergens (19). However, with high-throughput proteomics analysis technology, our group screened out eighty-four proteins (included Hsp60) of D. farinae, and we identified some of them as possible potential allergens (12). Moreover, the Hsp60 from D. farinae was successfully identified in this study. Our finding suggests a selectable way for screening dust-mite allergens, which also provides a new approach to identify new dust-mite allergens.

\section{Conclusions}

Taken together, the results in this study provide a solid foundation for further analyzing the allergenicity of $D$. farinae Hsp60 and its possible clinical application.

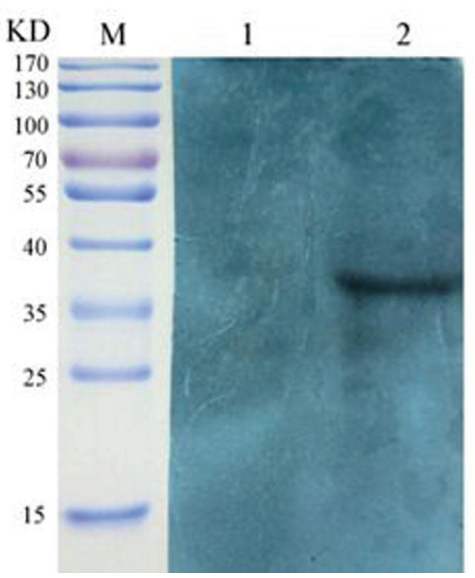

Figure 5. Western blot analysis of the recombinant Hsp60. Lane M: molecular mass standards; Lane 1: Expression product of pET-32a (+); Lane 2: Expression product of pET32a (+)-Hsp60. 


\section{Acknowledgement}

This research was supported by the science and technology plan projects from Guangdong Province (2013B031800022).

\section{Authors' contributions}

ZML and HJL carried out most of the experiments and wrote the manuscript. DXC has extensively revised the manuscript and the experimental design. JL, MSC, TC, QH, XDL, XML, XD, and HXS helped with the experiments. All the authors read and approved the final manuscript.

\section{Competing interests}

The authors declare that they have no competing interests.

\section{References}

1. Worldwide variations in the prevalence of asthma symptoms: the International Study of Asthma and Allergies in Childhood (ISAAC). Eur Respir J. 1998;12(2):315-335. doi:10.1183/090 31936.98.12020315

2. Aas K, Aaberg N, Bachert C, Schwicker D, Strachan D, Moerbeke DV, et al. European allergy white paper : allergic diseases as a public health problem in Europ. Braine-l'Alleud: UCB Institute of Allergy. 1997:117.

3. Maziak W, Behrens T, Brasky TM, Duhme H, Rzehak P, Weiland SK, et al. Are asthma and allergies in children and adolescents increasing? Results from ISAAC phase I and phase III surveys in Munster, Germany. Allergy. 2003;58(7):572-579. doi:10.1034/j.1398-9995.2003.00161.x

4. Franzese CB, Burkhalter NW. The patient with allergies. Med Clin North Am. 2010;94(5):891-902. doi:10.1016/j. mona.2010.05.006

5. Dust mite allergens and asthma--a worldwide problem. J Allergy Clin Immunol. 1989;83(2 Pt 1):416-427. doi:10.1016/00916749(89)90128-0

6. Custovic A, Simpson A, Woodcock A. Importance of indoor allergens in the induction of allergy and elicitation of allergic disease. Allergy. 1998;53(48 Suppl):115-120. doi:10.1111/j.1398-9995.1998.tb05011.x

7. Arlian LG. Mites are ubiquitous: are mite allergens, too? Ann Allergy Asthma Immunol. 2000;85(3):161-163. doi::10.1016/ S1081-1206(10)62461-1

8. Arlian LG, Platts-Mills TA. The biology of dust mites and the remediation of mite allergens in allergic disease. J Allergy Clin Immunol. 2001;107(3 Suppl):S406-413. doi:10.1067/ mai.2001.113670

9. Voorhorst R, Spieksma FTM, Varekamp H, Leupen MJ, Lyklema AW. The house-dust mite (Dermatophagoides pteronyssinus) and the allergens it produces. Identity with the house-dust allergen. J Allergy. 1967;39(6):325-339.

10. Bessot JC, Pauli G. Mite allergens: an overview. Eur Ann Allergy Clin Immunol. 2011;43(5):141-156. doi:10.1016/j.rmr. 2011.02.006. Epub 2011 Mar 24.

11. Bessot JC, Pauli G. [House dust mites allergens]. Rev Mal Respir. 2011;28(4):475-495.

12. Chen DX, Zhou PK, Ran PX. Screening and identification of dominant allergens of Dermatophagoides pteronyssinus and Dermatophagoides farina. China Tropical Medicine. 2009;9(2):232-234

13. Sambrook J, Russell DW. Molecular Cloning: A Laboratory Manual. Beijing: Science Press; 2002: 1228-1255, 1723-1726.

14. Young RA. Stress proteins and immunology. Annu Rev Immunol. 1990;8:401-420. doi:10.1146/annurev.iy.08.040190.002153

15. Kanamura HY, Hancock K, Rodrigues V, Damian RT. Schistosoma mansoni heat shock protein 70 elicits an early humoral immune response in S. mansoni infected baboons. Mem Inst Oswaldo Cruz. 2002;97(5):711-716. doi:10.1590/ S0074-02762002000500022

16. Zhang M, Hisaeda H, Kano S, Matsumoto Y, Hao YP, Looaresuwan S, et al. Antibodies specific for heat shock proteins in human and murine malaria. Microbes Infect. 2001;3(5):363367. doi:10.1016/S1286-4579(01)01391-0

17. Aki T, Fujikawa A, Wada T, Jyo T, Shigeta S, Murooka Y, et al. Cloning and expression of cDNA coding for a new allergen from the house dust mite, Dermatophagoides farinae: homology with human heat shock cognate proteins in the heat shock protein 70 family. $J$ Biochem. 1994;115(3):435-440. doi:10.1093/ oxfordjournals.jbchem.a124356

18. Liu HJ, Hong Q, Luo XD, Li XM, Ding X, Shen HX, et al. Dermatophagoides farinaeHsp60-DNASequencing, cloning and prokaryotic expression. Chinese J Zoonoses. 2015;31(2):154157. doi:10.3969/cjz.j.issn.1002-2694.2015.02.013

19. Puerta L, Caraballo L, Fernandez-Caldas E, Avjioglu A, Marsh DG, Lockey RF, et al. Nucleotide sequence analysis of a complementary DNA coding for a Blomia tropicalis allergen. J Allergy Clin Immunol. 1996;98(5 Pt 1):932-937. doi:10.1016/ s0091-6749(96)80009-1 